\title{
Optimal Insurance Under the Insurer's VaR Constraint
}

\author{
Chunyang Zhou and Chongfeng $\mathrm{Wu}$ \\ Financial Engineering Research Center, Shanghai Jiao Tong University, No. 535, Fahuazhen \\ Road, Shanghai, China. \\ E-mails: zcymagic@sjtu.edu.cn; cfwu@sjtu.edu.cn
}

In this paper, we impose the insurer's Value at Risk (VaR) constraint on Arrow's optimal insurance model. The insured aims to maximize his expected utility of terminal wealth, under the constraint that the insurer wishes to control the VaR of his terminal wealth to be maintained below a prespecified level. It is shown that when the insurer's VaR constraint is binding, the solution to the problem is not linear, but piecewise linear deductible, and the insured's optimal expected utility will increase as the insurer becomes more risk-tolerant. Basak and Shapiro (2001) showed that $\mathrm{VaR}$ risk managers often choose larger risk exposures to risky assets. We draw a similar conclusion in this paper. It is shown that when the insured has an exponential utility function, optimal insurance based on VaR constraint causes the insurer to suffer larger losses than optimal insurance without insurer's risk constraint.

The Geneva Risk and Insurance Review (2009) 34, 140-154. doi:10.1057/grir.2009.3

Keywords: optimal insurance; value at risk; risk constraint

\section{Introduction}

When faced with the uncertainty of loss, the insured can buy an insurance contract and transfer some risk to the insurer, who agrees to give him some payments when losses occur to him. There is a trade-off between the benefit of receiving indemnity and the cost of insurance premium. A problem arises about how a risk-averse insured balances the trade-off and chooses his optimal insurance so as to make him well off in the future.

The optimal insurance decision is usually determined by the maximization of the insured's expected utility or the minimization of the residual risk. Arrow (1963) showed that full coverage above a fixed deductible is optimal for a utility-maximizing individual. Under Arrow's framework, Raviv (1979), Deprez and Gerber (1985), Young (1999), Promislow and Young (2005), Zhou et al. (2008) used different insurance premium principles to study the optimal insurance problem. Instead of maximizing the expected utility of terminal wealth, Gajek and Zagrodny (2000, 2004) consider the optimal reinsurance problem by minimizing the insurer's residual risk. 
Wang et al. (2005) and Huang (2006) embedded the insured's Value at Risk (VaR) constraint into the optimal insurance problem. When an insurer offers the insured an insurance contract, he will take on the insured's risk and thus will be faced with some risk exposures. It is important for the insurer to control his risk exposure when he designs an optimal insurance contract. Zhou and $\mathrm{Wu}$ (2008) considered the optimal insurance problem under the insurer's expected loss constraint. Namely, the insured aims to maximize the expected utility of his terminal wealth, under the constraint that the expected loss of the insurer's terminal wealth is maintained below some prespecified level.

Value at Risk (VaR) is a standard risk measure for financial risk management, and is widely used in practice. VaR is defined as the "possible maximum loss over a given holding period within a fixed confidence level," where the confidence level is always set a relatively large value, such as 99 percent or 95 percent. In this paper, we extend the work of Zhou and $\mathrm{Wu}$ (2008), and solve the optimal insurance problem under the insurer's VaR risk constraint. It is shown that when the insurer's VaR constraint is binding, the solution to the problem is not linear, but piecewise linear deductible, and the insured's optimal expected utility will increase as the insurer becomes more risk tolerant. Basak and Shapiro (2001) showed that VaR risk managers often choose larger risk exposures to risky assets. We draw a similar conclusion in this paper. It is shown that when the insured has an exponential utility function, optimal insurance based on VaR constraint causes the insurer to suffer larger losses than optimal insurance without insurer's risk constraint.

The rest of paper is organized as follows. In the next section, we embedded the insurer's VaR constraint into Arrow's optimal insurance model. Following Raviv (1979) and Gollier (1987), we solve the problem via two steps. In the first step, we solve the optimal insurance problem with a fixed premium. Suppose when the premium is fixed as $\pi$, the optimal insurance is $I^{*}(x ; \pi)$. Then in the second step, we deal with the problem of finding the optimal $\pi^{*}$, thus completing the determination of the optimal insurance. In the next following section we deal with the first step, and in the subsequent section we proceed to deal with the second step. The penultimate section provides a numerical example where the insured has an exponential risk preference, and the losses are exponentially distributed. The final section concludes the paper.

\section{Model}

Consider a von-Neumann Morgenstern insured with an initial wealth $W_{1}$, will suffer a random loss $X$, a nonnegative continuous random variable defined on the probability space $(\Omega, F, P)$. The insured transfers part of his risk to an insurer, who agrees to give him a payment $I(x)$ when a loss $x$ occurs to him. 
$I(x)$ is always called the insurance policy or the coverage function in the literature, which satisfies $0 \leqslant I(x) \leqslant x$ for all $x \geqslant 0$. Let $I$ be the set of insurance policies that satisfy the condition. The insurance premium is assumed to be a function of the expected benefit received by the insured $h(E I)$, where $h(x)$ is a strictly increasing function of $x$ with $h(0)=0$, and $E I=\int{ }_{\Omega} I(X(\omega)) P(d \omega)$. The insurer will be faced with some risk exposures if he accepts the insurance contract, and he wishes to control the VaR of his terminal wealth to be maintained below some prespecified level. Suppose the insurer has an initial wealth $W_{2}$, then the constraint can be written as:

$$
\operatorname{Pr}\left(W_{2}-I+h(E I) \geqslant \underline{W}\right) \geqslant 1-\alpha
$$

where the floor $\underline{W}$ and the probability level $\alpha \in[0,1)$ are exogenously specified. Since the insurer behaves more risk tolerant as $\alpha$ increases, $\alpha$ can represent the insurer's risk tolerance level.

Let $u(x)$ be the insured's utility function, which satisfies $u^{\prime}>0$ and $u^{\prime \prime}<0$. The optimal insurance problem under the insurer's VaR risk constraint can be written as

$$
\begin{array}{ll}
\max _{I \in I} & E u\left(W_{1}-X+I-h(E I)\right) \\
\text { s.t. } & \operatorname{Pr}\left(W_{2}-I+h(E I) \geqslant \underline{W}\right) \geqslant 1-\alpha
\end{array}
$$

Clearly, the difference between Model (2) and Arrow model in Arrow (1963) is the insurer's risk constraint. It proves that the solution to the Arrow model can be written as $I^{*}(x)=(x-d)^{+}=\max (x-d, 0)$ (see Promislow and Young, 2005, for more discussions and a simplified proof). Therefore, if $I^{*}(x)$ satisfies the insurer's risk constraint, it is the solution to the problem (2) as well. However, when $I^{*}(x)$ does not satisfy the constraint, the insured will change his insurance policy to bind the insurer's risk constraint.

We follow Raviv (1979) and Gollier (1987), and solve the problem via two steps. In the next section, we first solve the optimal insurance problem with a fixed premium. Suppose when the premium is $\pi$, the insured's optimal insurance is $I^{*}(x ; \pi)$, which is a function of $\pi$. In the next following section, we proceed to determine the optimal $\pi^{*}$, thus completing the determination of the solution to the optimization problem (2).

\section{The optimal insurance with a fixed premium}

In this section, we keep the insurance premium fixed as $\pi$ and consider the following optimization problem:

$$
\max _{I} E u\left(W_{1}-X+I-\pi\right)
$$




$$
\begin{gathered}
\text { s.t. } E I=h^{-1}(\pi) \\
\operatorname{Pr}(I \leqslant \tau) \geqslant 1-\alpha \\
0 \leqslant I \leqslant X
\end{gathered}
$$

where $\tau=W_{2}+\pi-\underline{W}$, and $h^{-1}($.$) is the inverse function of h($.$) . Since h($.$) is a$ strictly increasing function, its inverse function $h^{-1}($.$) exists, and is a strictly$ increasing function as well.

For $0 \leqslant I \leqslant X$, it is easy to show that when $\pi>h(E X)$ or $\pi<0$, constraint (3b) will never be satisfied. Let $\bar{x}$ be such that $\operatorname{Pr}(X \leqslant \bar{x})=1-\alpha$. Then if $\tau>\bar{x}$, for $I \leqslant X$ we always have $\operatorname{Pr}(I \leqslant \tau) \geqslant \operatorname{Pr}(X \leqslant \bar{x})=1-\alpha$. Moreover, if $\tau<0$, we always have $\operatorname{Pr}(I \leqslant \tau)=0$. Therefore, when $\tau<0$, constraint (3c) will never be satisfied; and when $\tau \geqslant \bar{x}$, constraint (3c) will not be binding. In the following, we assume $0 \leqslant \pi \leqslant h(E X)$ and $\tau \geqslant 0$, and let $\Pi$ denote the collections of $\pi$ which satisfy the conditions.

When $\pi \in \Pi$, we have the following proposition about the solution to the optimization problem (3).

Proposition 1 Suppose $\pi \in \Pi$, and let $\bar{x}$ be such that $\operatorname{Pr}(X \leqslant \bar{x})=1-\alpha$. Then the solution to the optimization problem (3) can be written as

$$
I^{*}=\left\{\begin{array}{lll}
(x-d)^{+} & \text {if } & x \leqslant d+\tau \\
\tau & \text { if } & d+\tau<x \leqslant \bar{x} \\
x-d & \text { if } & x>\bar{x}
\end{array}\right.
$$

where $d \geqslant 0$ satisfies $E I^{*}=h^{-1}(\pi)$.

The proof can be found in Appendix A.

Thus from Proposition 1, we can conclude that the solution to the problem (3), if it exists, takes one of the two following forms:

$$
\begin{gathered}
I_{1}^{*}(x)=(x-d)^{+} \\
I_{2}^{*}(x)=\left\{\begin{array}{lll}
(x-d)^{+} & \text {if } & x \leqslant d+\tau \\
\tau & \text { if } & d+\tau<x \leqslant \bar{x} \\
x-d & \text { if } & x>\bar{x}
\end{array}\right.
\end{gathered}
$$

where the risk constraint (3c) is binding for $I_{2}^{*}$, and is not binding for $I_{1}^{*}$. It is clear that $I_{1}^{*}$ is the solution to the Arrow model with a fixed premium. If $I_{1}^{*}$ satisfies the insurer's risk constraint (3c), then $I_{1}^{*}$ solves the optimization 
144

problem (3). Otherwise, the insured will change his insurance policy to bind the insurer's risk constraint. In this case, we have the following proposition about the impact of the insurer's risk constraint on the insured's optimal choice of insurance policy.

Proposition 2 Suppose the solution to the problem (3) is $I_{2}^{*}(x)$ as Eq. (6). Without the insurer's risk constraint (3c), the solution is $I_{1}^{*}(x)=\left(x-d^{\prime}\right)^{+}$. Then we have $d<d^{\prime}<\bar{x}-\tau$.

Proof Since $I_{2}^{*}$ is the solution to the problem (3), and $I_{1}^{*}$ is not, from Eq. (6) it is easy to show that $(x+\tau-\bar{x})^{+} \leqslant I_{2}^{*}(x) \leqslant(x-d)^{+}$. Since $\operatorname{Pr}\left(I_{2}^{*}(x)<\right.$ $\left.(x-d)^{+}\right)>0$ we have $E\left(I_{2}^{*}\right)<E(X-d)^{+}$. Therefore if $d \geqslant d^{\prime}$, we have

$$
E\left(I_{2}^{*}\right)<E(X-d)^{+} \leqslant E\left(X-d^{\prime}\right)^{+}=E\left(I_{1}^{*}\right)
$$

which contradict the condition that $E\left(I_{1}^{*}\right)=E\left(I_{2}^{*}\right)=h^{-1}(\pi)$. Similarly we can prove $d^{\prime}<\bar{x}-\tau$.

Proposition 2 states that when $I_{1}^{*}(x)=\left(x-d^{\prime}\right)^{+}$does not satisfy the insurer's risk constraint, the deductible of the optimal insurance $I_{2}^{*}$ is less than $d^{\prime}$. Figure 1 depicts the curves of $I_{1}^{*}$ and $I_{2}^{*}$.

Figures 2 and 3 depict the impact of the insurer's VaR constraint on the insurer's and insured's terminal wealth, respectively. In Figure 2, $W_{2}\left(I_{1}^{*}\right)$ and $W_{2}\left(I_{2}^{*}\right)$ represent the insurer's terminal wealth under $I_{1}^{*}$ and $I_{2}^{*}$, respectively.

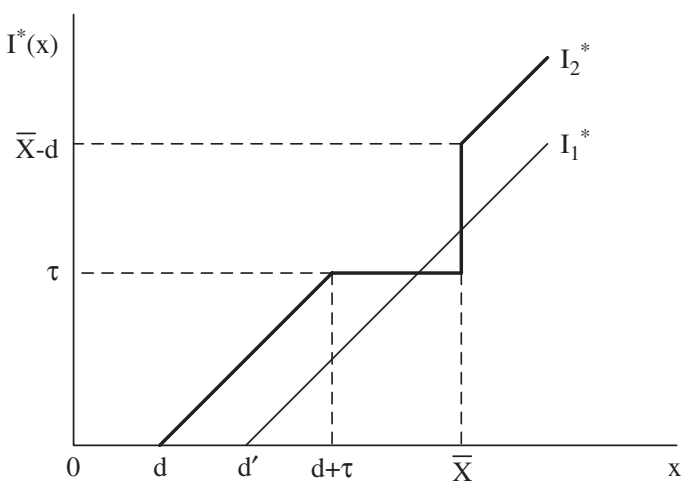

Figure 1. The impact of the insurer's risk constraint on the optimal policy. 


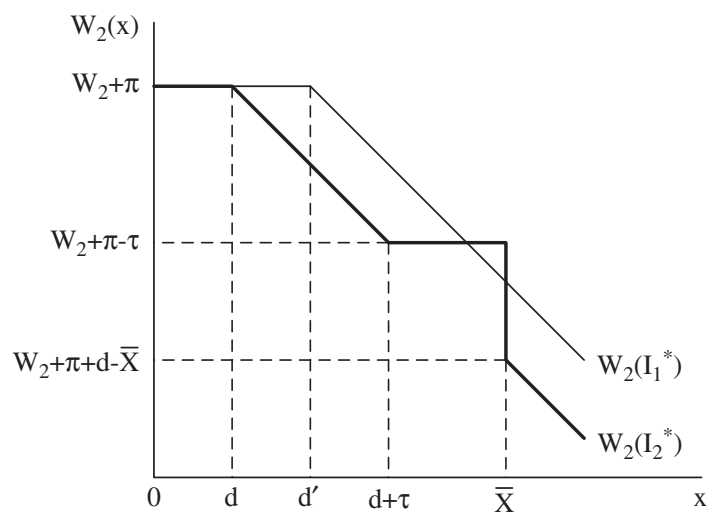

Figure 2. The impact of the insurer's risk constraint on the insurer's terminal wealth.

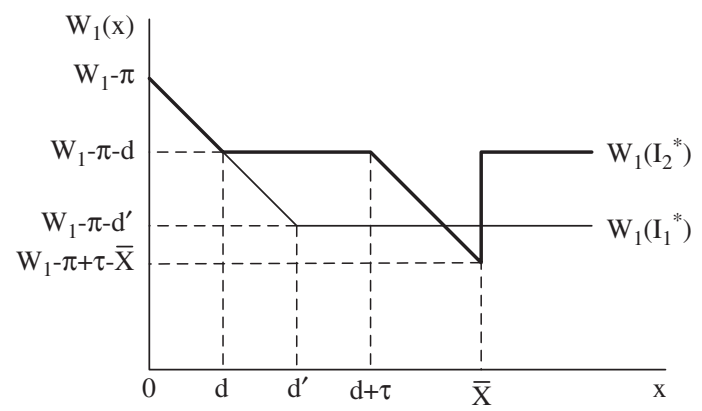

Figure 3. The impact of the insurer's risk constraint on the insured's terminal wealth.

Similarly in Figure 3, $W_{1}\left(I_{1}^{*}\right)$ and $W_{1}\left(I_{2}^{*}\right)$ represent the insured's terminal wealth under $I_{1}^{*}$ and $I_{2}^{*}$, respectively. It can be seen from Figure 2 that $I_{2}^{*}$ makes the insurer suffer more when large losses occur. Figure 3 shows that $I_{2}^{*}$ makes the insured better off when the loss is beyond $\bar{x}$.

\section{Determination of the optimal insurance}

In the previous section, the insurance premium is assumed to be fixed, and $d$ is a function of $\pi$. In this section, we proceed to determine the optimal premium, or the optimal $d$, thus completing the determination of the optimal insurance policy. 
146

Proposition 3 The solution to the optimization problem (2) takes one of two forms as Eqs (5) and (6). If $I_{1}^{*}$ is the optimal solution, where $d \in(0,+\infty)$, then $I_{1}^{*}$ shall satisfy

$$
\begin{gathered}
u^{\prime}\left(W_{1}-d-h\left(E I_{1}^{*}\right)\right) \\
=h^{\prime}\left(E I_{1}^{*}\right) E\left[u^{\prime}\left(W_{1}-X+I_{1}^{*}-h\left(E I_{1}^{*}\right)\right)\right] \\
\quad W_{2}-\underline{W}+h\left(E I_{1}^{*}\right) \geqslant(\bar{x}-d)^{+}
\end{gathered}
$$

If $I_{2}^{*}$ is the optimal solution, where $d, \tau \in(0,+\infty)$ and $d+\tau \leqslant \bar{x}$, then $I_{2}^{*}$ shall satisfy

$$
\begin{gathered}
u^{\prime}\left(W_{1}-d-h\left(E I_{2}^{*}\right)\right)\left[1-h^{\prime}\left(E I_{2}^{*}\right)+h^{\prime}\left(E I_{2}^{*}\right) F(d)\right] \\
=h^{\prime}\left(E I_{2}^{*}\right) E\left[u^{\prime}\left(W_{1}-X-h\left(E I_{2}^{*}\right)\right) 1_{\{X \leqslant d\}}\right] \\
W_{2}-\underline{W}+h\left(E I_{2}^{*}\right)=\tau
\end{gathered}
$$

where $F($.$) is the cumulative distribution function of X$. Moreover, let $V=E u\left(W-X+I_{2}^{*}-h\left(E I_{2}^{*}\right)\right)$ be the insured's optimal expected utility, then we have $\partial V / \partial \bar{x} \leqslant 0$. Since $\bar{x}$ is a decreasing function of $\alpha$, the insured's optimal expected utility will increase if the insurer's risk tolerance $\alpha$ increases.

The proof can be found in Appendix B.

Thus with the help of Proposition 3, we can solve the problem (2). Note that as well as Eq. (7), Eq. (9) can be interpreted economically, where the left-hand side is the marginal utility benefit of receiving additional indemnity, and the right-hand side represents the marginal utility cost of paying the corresponding additional premium. Since

$$
\begin{aligned}
E u^{\prime}\left(W_{1}-X-h\left(E I_{1}^{*}\right)\right)= & u^{\prime}\left(W_{1}-d-h\left(E_{1}^{*}\right)\right)(1-F(d)) \\
& +E\left[u^{\prime}\left(W_{1}-X-h\left(E I_{1}^{*}\right)\right) 1_{\{X \leqslant d\}}\right]
\end{aligned}
$$

Eq. (7) can also be written as

$$
\begin{gathered}
u^{\prime}\left(W_{1}-d-h\left(E I_{1}^{*}\right)\right)\left[1-h^{\prime}\left(E I_{1}^{*}\right)+h^{\prime}\left(E I_{1}^{*}\right) F(d)\right] \\
=h^{\prime}\left(E I_{1}^{*}\right) E\left[u^{\prime}\left(W_{1}-X-h\left(E I_{1}^{*}\right)\right) 1_{\{X \leqslant d\}}\right]
\end{gathered}
$$

which has a similar format as Eq. (9).

Proposition 3 states that if the insurer's risk constraint (3c) is binding, the insured will adopt $I_{2}^{*}$ as his optimal insurance, and his optimal expected utility 
will increase if the insurer's risk tolerance increases. When the insurer's risk tolerance increases to a level high enough to make $I_{1}^{*}$ satisfy the risk constraint, then the insured would adopt $I_{1}^{*}$ instead of $I_{2}^{*}$ as his optimal insurance policy. Now the insured's optimal expected utility will remain fixed even if the insurer increases his risk tolerance.

In the following proposition, we prove that when the insured has an exponential preference, the imposition of VaR constraint helps the insured transfer more of his large losses to the insurer.

Proposition 4 Suppose the insurer's utility is given by the exponential function as $u(W)=a-b e^{-R W}$, where $b, R>0$ and $R$ is the (constant) absolute risk aversion parameter. Let $d, \tau \geqslant 0$ be the solution to Eq. (9) and Eq. (10), and $d^{\prime}$ be the solution to Eq. (7). Then when $h^{\prime \prime}=0$ we have $d=d^{\prime}$; when $h^{\prime \prime}>0$ we have $d \leqslant d^{\prime}$.

The proof can be found in Appendix C.

Actually $h^{\prime \prime} \geqslant 0$ is a commonly used assumption in the literature (see Raviv, 1979). Proposition 4 says when the insured has an exponential preference, and the insurance premium is given by the expected value principle, i.e., $h(E I)=(1+\theta) E I$, the inclusion of insurer's loss constraint does not change the optimal deductible of Arrow's model. Under general setting $h^{\prime \prime} \geqslant 0, d \leqslant d^{\prime}$ implies that compared to Arrow model, the optimal insurance under insurer's VaR constraint provides more coverage for larger losses.

\section{An example: The exponential utility and exponential loss distribution}

In this section, we provide a simple example to illustrate the calculation process of the optimal insurance. The insured's utility is assumed to be $u(W)=a^{-1}\left(1-e^{-a W}\right)$ where $a>0$ is the (constant) absolute risk aversion. Then we have $u^{\prime}(W)=e^{-a W}$. The premium principle is given by $h(E I)=(1+\theta) E(I)$ where $\theta>0$. Let $X$ be the random variable with p.d.f $f(x)=\lambda e^{-\lambda x}$, where $x \geqslant 0$ and $\lambda$ is a positive constant. Since $1-\alpha=\operatorname{Pr}(X \leqslant \bar{x})=1-e^{-\lambda \bar{x}}$, we have

$$
\bar{x}=-\lambda^{-1} \ln \alpha
$$

If $I_{1}^{*}(x)=(x-d)^{+}$is the optimal insurance, then from Eq. (C.1) we have

$$
\begin{array}{lll}
(1+\theta)\left(\lambda e^{-a d}-a e^{-\lambda d}\right) & =\lambda-a & \text { if } \lambda \neq a \\
(1+\theta)(1+\lambda d) e^{-\lambda d} & =1 & \text { if } \lambda=a
\end{array}
$$

If $d$ satisfies the insurer's risk constraint Eq. (8), then $I_{1}^{*}(x)=(x-d)^{+}$is the optimal insurance. 
Otherwise, let $I_{2}^{*}(x)$ be as Eq. (6), then we have

$$
\begin{aligned}
h\left(E I_{2}^{*}\right)= & (1+\theta)\left[\int_{d}^{d+\tau}(x-d) \lambda e^{-\lambda x} d x+\int_{d+\tau}^{\bar{x}} \tau \lambda e^{-\lambda x} d x\right. \\
& \left.+\int_{\bar{x}}^{+\infty}(x-d) \lambda e^{-\lambda x} d x\right] \\
= & (1+\theta) \lambda^{-1} e^{-\lambda d}\left(1-e^{-\lambda \tau}\right) \\
& +(1+\theta)\left(\bar{x}-d-\tau+\lambda^{-1}\right) e^{-\lambda \bar{x}}
\end{aligned}
$$

Substitute it into Eq. (10), and we can obtain

$$
\begin{aligned}
\tau= & W_{2}-\underline{W}+(1+\theta) \lambda^{-1} e^{-\lambda d}\left(1-e^{-\lambda \tau}\right) \\
& +(1+\theta)\left(\bar{x}-d-\tau+\lambda^{-1}\right) e^{-\lambda \bar{x}}
\end{aligned}
$$

Thus from Proposition 4 we can calculate $d$ by solving Eq. (12), and $\tau$ by solving Eq. (13).

Let $\lambda=0.5, \theta=0.2, a=0.1, W_{2}-W=5, \alpha=0.05$, from Eqs (11), (12) and (8) we have $\bar{x} \approx 5.99, d \approx 3.56, h\left(E I_{1}^{*}\right)=(1+\theta) \lambda^{-1} e^{-\lambda d} \approx 0.40$ and

$$
W_{2}-\underline{W}+h\left(E I_{1}^{*}\right)-(\bar{x}-d)^{+} \approx 2.97>0
$$

Therefore in this case the optimal insurance is $I_{1}^{*}=(x-3.56)^{+}$.

Now let $\alpha=0.01$ with other parameters unchanged, then from Eq. (11) we have $\bar{x} \approx 9.21$. From Eq. (14), $d=3.56$ does not satisfy the insurer's risk constraint. From Eq. (13), we can obtain $\tau \approx 5.40$. Therefore in this case the optimal insurance is

$$
I_{2}^{*}(x)= \begin{cases}0 & \text { if } x \leqslant 3.56 \\ x-3.56 & \text { if } 3.56<x \leqslant 8.97 \\ 5.4 & \text { if } 8.97<x \leqslant 9.21 \\ x-3.56 & \text { if } x>9.21\end{cases}
$$

\section{Conclusion}

In this paper, we impose the insurer's VaR constraint on the Arrow's optimal insurance model, and discuss its impact on the insured's optimal choice of his insurance policy. 
Undoubtedly, if the solution to the Arrow model satisfies the insurer's risk constraint, then adding the constraint to the Arrow model will not change the solution. The solution can be written as $I_{1}^{*}(x)=(x-d)^{+}$. Otherwise, the insured will make his insurance policy bind the insurer's risk constraint. It is shown that now the insured's optimal insurance, if it exists, can be written as

$$
I_{2}^{*}=\left\{\begin{array}{lll}
(x-d)^{+} & \text {if } & x \leqslant \tau+d \\
\tau & \text { if } & \tau+d<x \leqslant \bar{x} \\
x-d & \text { if } & x>\bar{x}
\end{array}\right.
$$

We show that now the insured's optimal expected utility will increase if the insurer increases his risk tolerance. Basak and Shapiro (2001) showed that VaR risk managers often choose larger risk exposures to risky assets. We draw a similar conclusion in this paper. It is shown that when the insured has an exponential utility function, optimal insurance based on VaR constraint causes the insurer to suffer larger losses than optimal insurance without insurer's risk constraint.

\section{Acknowledgements}

The authors thank the anonymous referee for his/her helpful comments. This work is supported by National Natural Science Foundation of China (No. 70831004).

\section{References}

Arrow, K.J. (1963) 'Uncertainty and the welfare economics of medical care', American Economic Review 53: 941-973.

Basak, S. and Shapiro, A. (2001) 'Value-at-risk-based risk management: Optimal policies and asset prices', Review of Financial Studies 14: 371-405.

Deprez, O. and Gerber, H.U. (1985) 'On convex principles of premium calculation', Insurance: Mathematics and Economics 4: 179-189.

Gajek, L. and Zagrodny, D. (2000) 'Insurers optimal reinsurance strategies', Insurance: Mathematics and Economics 27: 105-112.

Gajek, L. and Zagrodny, D. (2004) 'Optimal reinsurance under general risk measures', Insurance: Mathematics and Economics 34: 227-240.

Gollier, C. (1987) 'The design of optimal insurance contracts without the nonnegativity constraint on claims', Journal of Risk and Insurance 54: 314-324.

Huang, H.H. (2006) 'Optimal insurance contract under a value-at-risk constraint', The Geneva Risk and Insurance Review 30: 161-179.

Jehle, G. and Reny, P. (2000) Advanced Microeconomics Theory, 2nd ed. Boston: Addison Wesley.

Karatzas, I. and Shreve, S.E. (1998) Methods of Mathematical Finance, New York: SpringerVerlag.

Promislow, S.D. and Young, V.R. (2005) 'Unifying framework for optimal insurance', Insurance: Mathematics and Economics 36: 347-364.

Raviv, A. (1979) 'The design of an optimal insurance policy', American Economic Review 69: 84-96. 
Wang, C.P., Shyu, D. and Huang, H.H. (2005) 'Optimal insurance design under a Value-at-Risk framework', The Geneva Risk and Insurance Review 30: 161-179.

Young, V.R. (1999) 'Optimal insurance under Wang's premium principle', Insurance: Mathematics and Economics 25: 109-122.

Zhou, C. and Wu, C. (2008) 'Optimal insurance under the insurer's risk constraint', Insurance: Mathematics and Economics 42: 992-999.

Zhou, C., Wu, C., Zhang, S. and Huang, X. (2008) 'An optimal insurance strategy for an individual under an inter temporal equilibrium', Insurance: Mathematics and Economics 42: 255-260.

\section{About the Authors}

Chunyang Zhou is Lecturer of Antai College of Economics \& Management, Shanghai Jiao Tong University, China.

Chongfeng Wu is Professor of Antai College of Economics \& Management, Shanghai Jiao Tong University, China.

\section{Appendix A}

Proof When $d+\tau>\bar{x}$, we have $I^{*}(x)=(x-d)^{+}$from Eq. (4). Since $I^{*}(x)$ is the solution to Arrow model with fixed premium, to prove $I^{*}(x)=(x-d)^{+}$is the solution to the problem (3), it remains to show that $I^{*}(x)$ satisfies the insurer's risk constraint ( $3 \mathrm{c})$. For $\tau \geqslant 0$, we have

$$
\begin{aligned}
\operatorname{Pr}\left(I^{*} \leqslant \tau\right) & =\operatorname{Pr}\left((X-d)^{+} \leqslant \tau\right) \\
& =\operatorname{Pr}(X \leqslant d+\tau) \geqslant \operatorname{Pr}(X \leqslant \bar{x})=1-\alpha
\end{aligned}
$$

where the inequality follows from the fact that $d+\tau>\bar{x}$ implies $\{\omega: X(\omega) \leqslant d+\tau\} \supseteq\{\omega: X(\omega) \leqslant \bar{x}\}$.

When $d+\tau \leqslant \bar{x}$, from Eq. (4) we have $\operatorname{Pr}\left(I^{*} \leqslant \tau\right)=\operatorname{Pr}(X \leqslant \bar{x})=1-\alpha$. Therefore, in this case $I^{*}$ satisfies the insurer's risk constraint (3c) with equality. It remains to prove $I^{*}$ is the solution to the optimization problem (3) where the risk constraint is binding. To show this, we adopt the convex-duality approach (see Karatzas and Shreve, 1998, for example) and consider the following dual optimization problem:

$$
\begin{array}{ll}
\max _{y} & g(y)=u\left(W_{1}-x+y-\pi\right)-\lambda_{1} y+\lambda_{2} 1_{\{y \leqslant \tau\}} \\
\text { s.t. } & 0 \leqslant y \leqslant x
\end{array}
$$

where $\lambda_{1} \in \mathbb{R}, \lambda_{2}=u\left(W_{1}-d-\pi\right)-\lambda_{1}(\bar{x}-d)-u\left(W_{1}-\bar{x}+\tau-\pi\right)+\lambda_{1} \tau$, and $1_{\{y \leqslant \tau\}}$ is the indication function whose value is 1 when $y \leqslant \tau$ and is zero otherwise. 
Lemma 1 The solution to the optimization problem (15) can be written as

$$
y^{*}=\left\{\begin{array}{lll}
(x-d)^{+} & \text {if } & x \leqslant d+\tau \\
\tau & \text { if } & d+\tau<x \leqslant \bar{x} \\
x-d & \text { if } & x>\bar{x}
\end{array}\right.
$$

where $d=\left(W_{1}-\pi-v\left(\lambda_{1}\right)\right)^{+}, v($.$) is the inverse function of u^{\prime}($.$) . Moreover we$ always have $\lambda_{2} \geqslant 0$.

Proof Let $g_{1}(y)=u\left(W_{1}-x+y-\pi\right)-\lambda_{1} y$, and $g_{1}{ }^{\prime}(y)=u^{\prime}\left(W_{1}-x+y-\pi\right)-\lambda_{1}=0$, we have $y=x-\left[W_{1}-\pi-v\left(\lambda_{1}\right)\right]$. Since $g_{1}(y)$ is a strictly concave function of $y$, it is easy to show that $g_{1}(y)$, when $y \in[0, x]$, attains its maximum at $y_{1}=(x-d)^{+}$, where $d=\left(W_{1}-\pi-v\left(\lambda_{1}\right)\right)^{+}$. Therefore, $g(y)=g_{1}(y)+\lambda_{2} 1_{\{y \leqslant \tau\}}$ when $y \in[0, x]$ attains its maximum at either $y_{1}=(x-d)^{+}$or $y_{2}=\tau$.

To get the optimal solution to the dual problem (15), we consider the following three cases:

(1) $x \leqslant d+\tau$

In this case we have $y_{1}=(x-d)^{+} \leqslant \tau$ and

$$
g\left(y_{1}\right)=g_{1}\left(y_{1}\right)+\lambda_{2} \geqslant g_{1}(\tau)+\lambda_{2}=g(\tau)
$$

Thus $g(y)$ attains its maximum when $y \in[0, x]$ at $y^{*}=y_{1}=(x-d)^{+}$.

(2) $d+\tau<x \leqslant \bar{x}$

In this case we have $y_{1}=x-d>\tau, g\left(y_{1}\right)=u\left(W_{1}-d-\pi\right)-\lambda_{1}(x-d)$, and

$$
\begin{aligned}
g(\tau)= & u\left(W_{1}-x+\tau-\pi\right)-\lambda_{1} \tau+\lambda_{2} \\
= & u\left(W_{1}-x+\tau-\pi\right)+u\left(W_{1}-d-\pi\right) \\
& -\lambda_{1}(\bar{x}-d)-u\left(W_{1}-\bar{x}+\tau-\pi\right) \\
= & g\left(y_{1}\right)+u\left(W_{1}-x+\tau-\pi\right)+\lambda_{1} x \\
& -\left[u\left(W_{1}-\bar{x}+\tau-\pi\right)-\lambda_{1} \bar{x}\right]
\end{aligned}
$$

Let $G(x)=u\left(W_{1}-x+\tau-\pi\right)+\lambda_{1} x$. Since $x>d+\tau \geqslant W_{1}-\pi-v\left(\lambda_{1}\right)+\tau$ and $u^{\prime}(x)$ is a decreasing function, we have

$$
G^{\prime}(x)=\lambda_{1}-u^{\prime}\left(W_{1}-x+\tau-\pi\right) \leqslant \lambda_{1}-u^{\prime}\left(v\left(\lambda_{1}\right)\right)=0
$$

Therefore $G(x)$ is a decreasing function of $x$. For $x \leqslant \bar{x}$, we have $G(x) \geqslant G(\bar{x})$. Substituting it into Eq. (A.2), we have $g(\tau) \geqslant g\left(y_{1}\right)$ from Eq. (A.2). Meanwhile since $0 \leqslant \tau \leqslant x-d \leqslant x$, we have $g(y)$ when $y \in[0, x]$ attains its maximum at $y^{*}=\tau$.

(3) $x>\bar{x}$

Similar to case (2), it is easy to show that $g\left(y_{1}\right)>g(\tau)$ in this case. Since $x \geqslant d$, we have $g(y)$ when $y \in[0, x]$ attains its maximum at $y^{*}=x-d$. 
Finally, to show $\lambda_{2} \geqslant 0$, note that we have

$$
\begin{aligned}
\lambda_{2}= & u\left(W_{1}-(d+\tau)+\tau-\pi\right)+\lambda_{1}(d+\tau) \\
& -\left[u\left(W_{1}-\bar{x}+\tau-\pi\right)+\lambda_{1} \bar{x}\right] \geqslant 0
\end{aligned}
$$

where the inequality follows from $G(x)$ is a decreasing function of $x$ and $d+\tau \leqslant \bar{x}$.

Now let $d \geqslant 0$ be the solution to $E\left(I^{*}\right)=h^{-1}(\pi)$ and $\lambda_{1}=u^{\prime}\left(W_{1}-\pi-d\right)$. Applying Lemma 1 pointwise for all $x \geqslant 0$, it follows that Eq. (4) is the solution to the optimization problem.

$$
\begin{array}{ll}
\max _{I} & u\left(W_{1}-X+I-\pi\right)-\lambda_{1} I+\lambda_{2} 1_{\{I \leqslant \tau\}} \\
\text { s.t. } & 0 \leqslant I \leqslant X
\end{array}
$$

Let $J$ be any candidate coverage function which satisfies the constraints (3b, $3 c$, 3d), we have

$$
\begin{aligned}
E u( & \left.W_{1}-X+I^{*}-\pi\right)-E u\left(W_{1}-X+J-\pi\right) \\
= & E u\left(W_{1}-X+I^{*}-\pi\right)-\lambda_{1} h^{-1}(\pi)+\lambda_{2}(1-\alpha) \\
& -\left[E u\left(W_{1}-X+J-\pi\right)-\lambda_{1} h^{-1}(\pi)+\lambda_{2}(1-\alpha)\right] \\
& \geqslant E u\left(W_{1}-X+I^{*}-\pi\right)-\lambda_{1} E\left(I^{*}\right)+\lambda_{2} E\left(1_{\left\{I^{*} \leqslant \tau\right\}}\right) \\
& -\left[E u\left(W_{1}-X+J-\pi\right)-\lambda_{1} E(J)+\lambda_{2} E\left(1_{\{J \leqslant \tau\}}\right)\right] \geqslant 0
\end{aligned}
$$

where the first inequality follows from the fact that the constraint (3c) holds for $I^{*}$ with equality, while holding for $J$ with inequality.

\section{Appendix B}

Proof of Proposition 3:

Proof (1) When $I_{1}^{*}$ is the optimal solution, let

$$
U(d)=E u\left(W_{1}-X+(X-d)^{+}-h\left(E(X-d)^{+}\right)\right)
$$

From the first order condition $U^{\prime}(d)=0$, we have Eq. (7). Moreover for $I_{1}^{*}$ satisfies the risk constraint $(3 \mathrm{c})$ we have

$$
\operatorname{Pr}\left((X-d)^{+} \leqslant W_{2}+h\left(E(X-d)^{+}\right)-\underline{W}\right) \geqslant 1-\alpha
$$

Since $\alpha \in[0,1)$, we have $W_{2}+h\left(E(X-d)^{+}\right)-\underline{W} \geqslant 0$ and Eq. (B.1) can be written as

$$
\operatorname{Pr}\left(X \leqslant d+W_{2}+h\left(E(X-d)^{+}\right)-\underline{W}\right) \geqslant 1-\alpha
$$


Since $\operatorname{Pr}(X \leqslant \bar{x})=1-\alpha$, we can obtain

$$
d+W_{2}+h\left(E(X-d)^{+}\right)-\underline{W} \geqslant \bar{x}
$$

Thus we have proved the first conclusion.

(2) If $I_{2}^{*}$ is the solution to the problem (2), it is easy to show that $I_{2}^{*}$ satisfies the insurer's risk constraint with equality. We can calculate $d$ and $\tau$ by solving the following optimization problem:

$$
\begin{array}{ll}
\max _{d, \tau} & U(d, \tau)=E u\left(W_{1}-X+I_{2}^{*}-h\left(E I_{2}^{*}\right)\right) \\
\text { s.t. } & M(d, \tau)=h\left(E I_{2}^{*}\right)-\tau-\underline{W}+W_{2}
\end{array}
$$

Let $L(d, \tau)=U(d, \tau)-\lambda M(d, \tau)$, where $\lambda$ is the Lagrange multiplier. From the condition $\partial L / \partial d=0$, we have

$$
\begin{aligned}
& u^{\prime}\left(W_{1}-d-h\left(E I_{2}^{*}\right)\right)=h^{\prime}\left(E I_{2}^{*}\right) \\
& {\left[E u^{\prime}\left(W_{1}-X+I_{2}^{*}-h\left(E I_{2}^{*}\right)\right)+\lambda\right]}
\end{aligned}
$$

From the condition $\partial L / \partial \tau=0$, we have

$$
\begin{aligned}
& {[F(\bar{x})-F(d+\tau)] h^{\prime}\left(E I_{2}^{*}\right)} \\
& {\left[E u^{\prime}\left(W_{1}-X+I_{2}^{*}-h\left(E I_{2}^{*}\right)\right)+\lambda\right]} \\
& =\int_{d+\tau}^{\bar{x}} u^{\prime}\left(W_{1}-x+\tau-h\left(E I_{2}^{*}\right)\right) f(x) d x+\lambda
\end{aligned}
$$

From Eqs (B.2) and (B.3), by eliminating $\lambda$ we can obtain Eq. (7).

Moreover, from the envelope theorem (see Jehle and Reny, 2000, Appendix 2.4 for more details), we have

$$
\begin{aligned}
& \frac{\partial V}{\partial \bar{x}}=\frac{\partial L}{\partial \bar{x}}=f(\bar{x}) \\
& {\left[u\left(W_{1}-\bar{x}+\tau-h\left(E I_{2}^{*}\right)\right)-u\left(W_{1}-d-h\left(E I_{2}^{*}\right)\right)\right]} \\
& -f(\bar{x})(\tau+d-\bar{x}) u^{\prime}\left(W_{1}-d-h\left(E I_{2}^{*}\right)\right)
\end{aligned}
$$

Since $u($.$) is a concave function, we have$

$$
\begin{aligned}
& u\left(W_{1}-\bar{x}+\tau-h\left(E I_{2}^{*}\right)\right) \\
& -u\left(W_{1}-d-h\left(E I_{2}^{*}\right)\right) \\
& \leqslant(\tau+d-\bar{x}) u^{\prime}\left(W_{1}-d-h\left(E I_{2}^{*}\right)\right)
\end{aligned}
$$

Substituting it into Eq. (B.4), we have $\partial V / \partial \bar{x} \leqslant 0$. 


\section{Appendix C}

Proof of Proposition 4:

Proof From Eq. (9) and the exponential utility function, we have

$$
\begin{gathered}
b \operatorname{Re}^{-R\left(W-d-h\left(E I_{2}^{*}\right)\right)}\left[1-h^{\prime}\left(E I_{2}^{*}\right)+h^{\prime}\left(E I_{2}^{*}\right) F(d)\right] \\
=h^{\prime}\left(E I_{2}^{*}\right) \int_{0}^{d} b \operatorname{Re}^{-R\left(W-x-h\left(E I_{2}^{*}\right)\right)} d F(x)
\end{gathered}
$$

which can also be written as

$$
h^{\prime}\left(E I_{2}^{*}\right)\left[\int_{0}^{d} e^{-R(d-x)} d F(x)+1-F(d)\right]=1
$$

Similarly from Eq. (7) and the exponential preference, we have

$$
\begin{aligned}
& h^{\prime}\left(E I_{1}^{*}\right)\left[\int_{0}^{d^{\prime}} b \operatorname{Re}^{-R\left(W-x-h\left(E I_{1}^{*}\right)\right)} d F(x)\right. \\
& \left.+b \operatorname{Re}^{-R\left(W-d^{\prime}-h\left(E I_{1}^{*}\right)\right)}(1-F(d))\right] \\
& =b \operatorname{Re}^{-R\left(W-d^{\prime}-h\left(E I_{1}^{*}\right)\right)}
\end{aligned}
$$

which can be simplified as

$$
h^{\prime}\left(E I_{1}^{*}\right)\left[\int_{0}^{d^{\prime}} e^{-R\left(d^{\prime}-x\right)} d F(x)+1-F\left(d^{\prime}\right)\right]=1
$$

Let $G(z)=\int_{0}^{z} e^{-R(z-x)} d F(x)+1-F(z)$. We know $G(z)$ is a decreasing function since $G^{\prime}(z)=-R \int_{0}^{z} e^{-R(z-x)} d F(x) \leqslant 0$. Therefore if $h^{\prime \prime}=0, h^{\prime}(x)$ is a constant for any $x$. From Eqs (C.1) and (C.2) we have $d=d^{\prime}$.

If $h^{\prime \prime}>0$ and $d>d^{\prime}$, it easy to show that $E I_{2}^{*}<E I_{1}^{*}$ and $h^{\prime}\left(E I_{2}^{*}\right)<h^{\prime}\left(E I_{1}^{*}\right)$. $G(d)<G\left(d^{\prime}\right)$ can be obtained since $G(z)$ is a decreasing function. For both $h^{\prime}($. and $G\left(\right.$.) are positive functions, we have $h^{\prime}\left(E I_{2}^{*}\right) G(d)<h^{\prime}\left(E I_{1}^{*}\right) G\left(d^{\prime}\right)$. However, from Eqs (C.1) and (C.1) we know $h^{\prime}\left(E I_{2}^{*}\right) G(d)=h^{\prime}\left(E I_{1}^{*}\right) G\left(d^{\prime}\right)$. The contraction implies that $d \leqslant d^{\prime}$. 